\title{
Magnetization in two-dimensional electron gas in a perpendicular magnetic field: the roles of edge states and spin-orbit coupling
}

\author{
Zhigang Wang, ${ }^{1}$ Wei Zhang, ${ }^{1}$ and Ping Zhang ${ }^{1,2}$, * \\ ${ }^{1}$ LCP, Institute of Applied Physics and Computational Mathematics, \\ P.O. Box 8009, Beijing 100088, People's Republic of China \\ ${ }^{2}$ Center for Applied Physics and Technology, Peking University, Beijing 100871, People's Republic of China
}

\begin{abstract}
We study the de Haas-van Alphen (dHvA) oscillations in the magnetization of a two-dimensional electron gas (2DEG) under the influence of the edge states and/or the Rashba spin-orbit interaction (SOI). The boundaries of the systems lift partially the degeneracies of Landau levels (LL's) and the resulting edge states lead to the changes of both the center and the amplitude of the sawtoothlike magnetization oscillation. The SOI mixes the spin-up and spin-down states of neighboring LL's into two unequally spaced energy branches. The inclusion of SOI changes the well-defined sawtooth pattern of the dHvA oscillations in the magnetization. The weaker the magnetic field is, the larger is the change of the dHvA oscillations due to the edge effect and/or the spin-orbit coupling. Some theoretical results are compared with the experimental data.
\end{abstract}

PACS numbers: 71.10.Ca, 73.20.At, 72.15.Gd

\section{INTRODUCTION}

The physics of two-dimensional electron gas (2DEG) in the presence of a perpendicular magnetic field reveals a rich variety of remarkable phenomena, for example the integer and fractional quantum Hall effects (QHEs). Recently, the magnetization property of $2 \mathrm{DEG}$ at low temperature and in a strong perpendicular magnetic field has attracted extensive interest. It is due to the fact that the magnetization is particularly suited to investigate the electronic ground-state properties and the density of states (DOS) of 2DEG by its minimal perturbation to the system 1 . On the experimental side, the measurement of the magnetization keeps a most challenging task due to weak signal of the magnetization. A variety of techniques, such as dc superconducting quantum interference device (SQUID) magnetometers $2.3,4,5$, picking up coils lithographed above the gate ${ }^{\underline{6}}$, torque magnetometers $7,8,9,10,11,12$, torsional magnetometer with optical angular detection $13,14,15$, and micromechanical cantilever magnetometers $16,17,18,19,20,21,22$, have been developed to observe the magnetization oscillations, i.e., the de Haas-van Alphen (dHvA) effect in high-mobility 2DEG. Clear sawtooth dHvA oscillations in the magnetization have been observed for LLs of filling factors up to $52^{10}$. More recently, a novel method has been used by Prus et al $\stackrel{23}{\underline{20}}$ and Shashkin et al $\stackrel{24}{*}$ to measure the spin magnetization of 2DEG in silicon metal-oxide-semiconductor field-effect transistors (MOSFETs). This method entails modulating the magnetic field with an auxiliary coil and measuring the imaginary (out-of-phase) component of the ac current induced between the gate and the 2DEG system, which is proportional to $\partial \mu / \partial B$ (where $\mu$ is the chemical potential and $B$ is the magnetic field). Using the Maxwell relation, $\partial \mu / \partial B=-\partial \mathcal{M} / \partial \mathcal{N}$, one can then obtain the magnetization $\mathcal{M}$ by integrating the induced current over the electron density, $\mathcal{N}$. Pauli spin susceptibility has been observed to behave critically near the $2 \mathrm{D}$ metalinsulator transition, in agreement with previous transport measurements 25,26 . With the similar method, Anis-

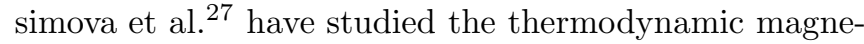
tization of a low-disordered, strongly correlated 2DEG in silicon MOSFETs in perpendicular and tilted magnetic fields. By measuring $\partial \mu / \partial B$ at noninteger filling factors, they have directly determined the spectrum characteristics without any fitting procedures or parameters.

On the theoretical side, extensive studies of dHvA oscillations in the magnetization of 2DEG have also been carried out 28,29,30,31,32,33,34. In particular, Bremme et al ${ }^{30}$ have investigated the influence of the edge current on the dHvA oscillations in the magnetization of a 2DEG using a spinless single-particle approach. Sharapov et al $\stackrel{31}{=}$ have extensively discussed the dHvA oscillations of the magnetization in planar system with the Dirac-like spectrum of quasiparticle excitation. In addition, the magnetization oscillations as a function of the magnetic field has also been theoretically studied in quantum dot systems $32,33,34$. However, to our knowledge there are no detailed treatments of the influence of edge states and the SOI on the magnetization in 2D systems. In this paper, we study systematically the thermodynamic magnetization of a 2DEG system with edge states and SOI. In particular, we address the effects of SOI and edge states on the Landau level (LL) structure, the chemical potential, and the magnetization and its susceptibility to strong magnetic field. Quantum oscillations in the magnetization of a 2DEG are well known to be characterized by strictly $(1 / B)$-periodic sawtoothlike oscillations with an amplitude of 1 effective Bohr magneton $\mu_{B}\left(=e \hbar / 2 m^{*}\right.$ with $m^{*}$ the effective electron mass) per electron. We will show that the picture changes for the case in the presence of SOI and edge states. The degeneracy of LL's plays an important role in the formation of $\mathrm{dHvA}$ oscillations. The edge states lift partially the degeneracies of Landau levels and lead to the change of both the center and the amplitude of the sawtoothlike magnetization oscillation. 
The SOI mixes the spin-up and spin-down states of neighboring LL's into two unequally spaced energy branches. The inclusion of SOI changes the well-defined sawtooth behavior of the dHvA oscillations in the magnetization. These results may be found useful in the characterization of magnetic oscillations in two dimensional systems.

In Sec. II we review the exactly solvable cases of bulk 2DEG with or without Rashba SOI, and numerically solvable cases in the presence of both edge states and Rashba SOI. In Sec. III we present the results for the magnetization and the effects of SOI and/or edge states. The results on the magnetic susceptibility are presented in section IV. Some concluding remarks are given in Sec. V.

\section{ENERGY SPECTRUM FOR 2DEG}

We consider a 2DEG with the Rashba coupling in the $x-y$ plane of an area $L_{x} \times L_{y}$ subject to a perpendicular magnetic filed $\mathbf{B}=B \hat{z}$. The electrons are confined between 0 and $L_{y}$ in the $y$ direction by an infinite potential wall, and its wave function is periodic along the $x$ direction. We choose the Landau gauge $\mathbf{A}=-B y \hat{\mathbf{x}}$. The Hamiltonian for a single electron of spin- $1 / 2$ with a Rashba coupling is given by

$$
H_{0}=\frac{\vec{\Pi}^{2}}{2 m^{*}}+\frac{\lambda}{\hbar}\left(\Pi_{x} \sigma_{y}-\Pi_{y} \sigma_{x}\right)-\frac{1}{2} g_{s} \mu_{B} B \sigma_{z}+V(y),
$$

where $m^{*},(-e)$, and $g_{s}$ are the electron's effective mass, charge and effective magnetic factor respectively, $\mu_{B}$ is the Bohr magneton, $\vec{\Pi}=\vec{p}+e \vec{A} / c$ is the kinetic operator, $\lambda$ is the Rashba coupling, and $\sigma_{\alpha}$ are the Pauli matrices. The last term $V(y)$ is the lateral confining potential: $V(y)=0$ for $0 \leqslant y \leqslant L_{y}$ and infinite otherwise. Relevant quantities related to the magnetic field are the cyclotron frequency $\omega_{c}=e B / m^{*}$ and the magnetic length $l_{b}=\sqrt{\hbar / e B}$. The Rashba SOI in Eq. (1) stems from the structural inversion asymmetry (SIA) introduced by a heterojunction or by surface or external fields. In semiconductors with narrower energy gap (InGaAs, AlGaAs), this effect is expected to be stronger ${ }^{35}$. It has been shown experimentally that the Rashba SOI can be modified up to $50 \%$ by external gate voltages 36,37 .

Without considering the edge-state effect, the magnetization of a $2 \mathrm{D}$ spinless electron moving in a high perpendicular magnetic field is a 3D simplification that has been solved by Landau in a pioneering paper published in $1930^{38}$. In that (2D) case, each state is described by two quantum numbers $k$ and $n$. The quantum number $k$ denotes the $x$ component of the electron momentum (scaled by $\hbar)$ and is a constant of motion. The other quantum number $n$ being the Landau-level index describes different modes of a displaced linear oscillator of frequency $\omega_{c}$. The LL's are

$$
E_{n}=\left(n+\frac{1}{2}\right) \hbar \omega_{c}
$$

and the corresponding eigenstates are

$$
|n, k\rangle=\frac{1}{\sqrt{L_{x}}} e^{i k x} \frac{e^{-\left(y-y_{0}\right)^{2} / 2 l_{b}^{2}}}{\left(\sqrt{\pi} 2^{n} n ! l_{b}\right)^{1 / 2}} H_{n}\left(\frac{y-y_{0}}{l_{b}}\right),
$$

where $H_{n}(x)$ is the Hermite polynomial, and $y_{0}=l_{b}^{2} k$ is the center of the cyclotron orbit. The degeneracy $N_{L}$ of each Landau level per spin is given by $N_{L}=2 \pi S / l_{b}^{2}$ with $S=L_{x} L_{y}$ being the area of the sample. When the electron spin degrees of freedom is included but the edge-state effect and spin-orbit coupling are excluded, the eigenstate is characterized by three quantum numbers,

$$
|n, k, s\rangle=|n, k\rangle \chi_{s},
$$

where $s= \pm 1 / 2$ and $\chi_{ \pm 1 / 2}$ is the eigenstate of spin operator $\hat{s}_{z}$ with eigenvalues $\pm \hbar / 2$. In this case, the spin-split LL's are

$$
E_{n, s}=E_{n}-s g_{s} \mu_{B} B .
$$

Thus each LL is split into spin-up and spin-down branches. When the edge channels are furthermore included but the SOI is excluded 39.40 , the eigenstate is still a product state of spin and orbital degrees of freedom. However, the orbital part of the wave function is no longer a form of Hermite function due to the confinement along $y$ direction. In this case, the eigenstate is given by $|n, k, s\rangle=\frac{1}{\sqrt{L_{x}}} e^{i k x} \varphi_{n, y_{0}}(y) \chi_{s}$, where $\varphi_{n, y_{0}}(y)$ obeys

$\left[-\frac{p_{y}^{2}}{2 m^{*}}+\frac{1}{2} m^{*} \omega_{c}\left(y-y_{0}\right)^{2}+V(y)\right] \varphi_{n, y_{0}}(y)=\epsilon_{n}\left(y_{0}\right) \varphi_{n, y_{0}}(y)$.

This equation has been solved by MacDonald et al. through properly applying the boundary condition $\underline{40}$. The resulting eigenvalue spectrum of Eq. (6) has a form $\epsilon_{n}\left(y_{0}\right)=\left[\nu_{n}\left(y_{0}\right)+\frac{1}{2}\right] \hbar \omega_{c}$, where $\nu_{n}\left(y_{0}\right)$ is numerically obtained by requiring the wave function $\varphi_{n, y_{0}}(y)$ to vanish at the boundary $y=0, L_{y}$. In this case, the spin-split LL's are given by

$$
E_{n, s}\left(y_{0}\right)=\left[\nu_{n}\left(y_{0}\right)+\frac{1}{2}\right] \hbar \omega_{c}-s g_{s} \mu_{B} B,
$$

which as an example is illustrated in Fig. 1(a) as a function of the guiding center $y_{0}$.

On the other side, when the Rashba SOI is included while the edge-state effect is excluded, the spin-orbit coupling mixes the two spin components. In this case, the energies of the two branches of states (denoted by \pm ) are given by

$$
E_{n}^{ \pm}=\hbar \omega_{c}\left(n \pm \sqrt{(1-g)^{2}+8 n \eta^{2}}\right)
$$

for $n \geq 1$, where we have defined $g=g_{s} m^{*} / 2 m_{e}$ and the effective (dimensionless) Rashba coupling $\eta=\lambda m^{*} l_{b} / \hbar^{2}$. For $n=0$, there is only one single state with the energy 
$E_{0}^{+}=\hbar \omega_{c}(1-g)$, which is the same as the lowest Landau level without SOI. The corresponding eigenstates are given by

$$
\left|n, y_{0}, \pm\right\rangle=\left(\begin{array}{c}
\cos \theta_{n}^{ \pm}\left|n, y_{0}\right\rangle \\
i \sin \theta_{n}^{ \pm}\left|n-1, y_{0}\right\rangle
\end{array}\right)
$$

for $n \geq 1$, where the parameters $\theta_{n}^{ \pm}$are given by $\tan \theta_{n}^{ \pm}=-u_{n} \pm \sqrt{1+u_{n}^{2}}$ with $u_{n}=(1-g) / \sqrt{8 n} \eta$. For $n=0$, the single state is a product of the ground-state oscillator mode $|0, k\rangle$ and eigenstate $\chi_{1 / 2}$ of $\hat{s}_{z}$. Thus it is interesting to see that the ground state $(n=0)$ has the fully-polarized spin along the $z$ direction. In the excited states the spin is tilted with an expectation value of its $z$ component $\left\langle\sigma_{z}\right\rangle=\cos ^{2} \theta_{n}^{ \pm}-\sin ^{2} \theta_{n}^{ \pm}$that decreases as $\lambda$ and $n$ increase. A prominent feature is that the two branches of Landau levels $E_{n}^{+}$and $E_{n+1}^{-}$cross each other at the values of $\eta$ satisfying

$$
\sqrt{(1-g)^{2}+8 n \eta^{2}}+\sqrt{(1-g)^{2}+8(n+1) \eta^{2}}=2 .
$$

This degenerate behavior in the energy spectrum has been used to produce the resonant spin-Hall current ${ }^{41.42}$.

When both the edge states and the Rashba SOI are included, two interplayed mixing mechanisms occur. One is from the coupling between different confining orbital modes along the $y$ direction; the other is the mixing of the eigenstates $\chi_{1 / 2}$ and $\chi_{-1 / 2}$ of the spin operator $s_{z}$. As a result, the wave function for the final Hamiltonian (1) can be written in a general form ${ }^{43}$ :

$$
\Psi_{n}(x, y)=\frac{1}{\sqrt{L_{x}}} e^{i k x} \varphi_{n}(y) .
$$

Here $\varphi_{n}(y)$ are expanded in the basis of the infinite potential well,

$$
\varphi_{n}(y)=\sqrt{\frac{2}{L_{y}}} \sum_{m} \sin \left(\frac{\pi m}{L_{y}} y\right)\left(\begin{array}{c}
a_{m n} \\
b_{m n}
\end{array}\right),
$$

with $n$ being the Landau-level index and $m$ an integer. The Schrödinger equation $H_{0} \Psi_{n}=E_{n}\left(y_{0}\right) \Psi_{n}$ leads to the following equations for the spinors:

$$
\begin{aligned}
& {\left[A_{l \pm}-E_{n}\right]\left(\begin{array}{c}
a_{l n} \\
b_{l n}
\end{array}\right)} \\
& =\sum_{m}\left[i\left(F_{l m}+G_{l m}\right) \sigma^{-}+i\left(F_{l m}-G_{l m}\right) \sigma^{+}-M_{l m}\right]\left(\begin{array}{c}
a_{m n} \\
b_{m n}
\end{array}\right)
\end{aligned}
$$

where $\sigma^{ \pm}=\left(\sigma_{x} \pm i \sigma_{y}\right) / 2, \epsilon_{n}=E_{n} / \hbar \omega_{c}$. The other parameters in Eq. (13) are defined as

$$
\begin{aligned}
A_{l \pm} & =\left(\frac{\pi l}{2}\right)^{2}\left(\frac{l_{b}}{L_{y}}\right)^{2} \mp \frac{g}{2} \\
M_{l m} & =\frac{1}{\pi^{3}}\left(\frac{L_{y}}{l_{b}}\right)^{2} \int_{0}^{\pi} d \theta \sin (l \theta)\left(\theta-\theta_{0}\right)^{2} \sin (m \theta) \\
F_{l m} & =\frac{2 \eta}{\pi^{2}} \frac{L_{y}}{l_{b}} \int_{0}^{\pi} d \theta \sin (l \theta)\left(\theta-\theta_{0}\right) \sin (m \theta) \\
G_{l m} & =2 \eta \frac{l_{b}}{L_{y}} \int_{0}^{\pi} d \theta \sin (l \theta) \frac{\partial}{\partial \theta} \sin (m \theta)
\end{aligned}
$$
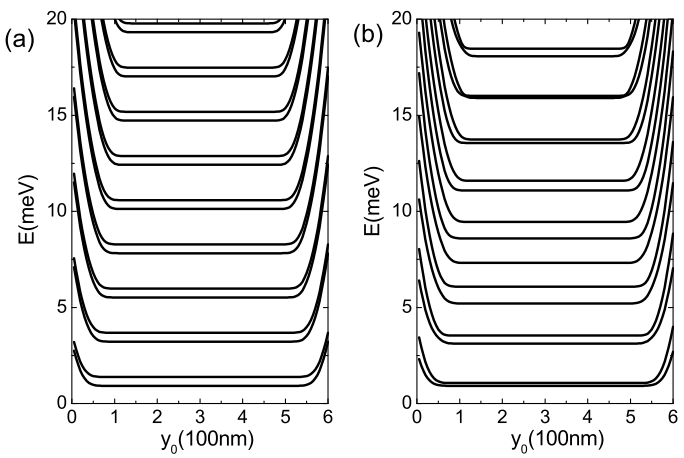

FIG. 1: The energy spectrum in units of meV versus the guiding center $y_{0}$ without (a) and with the Rashba spin-orbit couplings (b), respectively. In both figures, the parameters are chosen as $m^{*}=0.05 m_{e}, L=600 \mathrm{~nm}, B=1 \mathrm{~T}$, and $g_{s}=4$. The Rashba SOI strength in (b) is set as $\lambda=15 \mathrm{meV}$ nm.

where $\theta_{0}=y_{0} \pi / L_{y}$. We solve these equations in a truncated Hilbert space disregarding the states with energies higher than the cutoff energy. Typically we take a matrix Hamiltonian of dimension of a few hundred. We increase the size of the Hilbert space by a factor 2 and find no change in the results presented below. In all cases the width of the sample $L_{y}$ is taken large enough to have the cyclotron radius $r_{c}$ smaller than $L_{y} / 2$. The right and left edge states are then well separated in real space. A typical energy spectrum is shown in Fig. 1(b). For $y_{0} \simeq L_{y} / 2$ the states are equal to the bulk states, except for exponential corrections. The wave functions and the energy spectrum reproduces the above-discussed bulk results without edge states. As $y_{0}$ approaches the sample edge, the effect of the confining potential becomes important and it generates the $k$-dependent dispersion of the energy levels ${ }^{39}$, which has profound effects on magnetotransport and magnetization properties.

\section{THE MAGNETIZATION OF 2DEG}

We begin with a briefly review of the standard derivation of the 2DEG magnetization from the free energy. The magnetization density is the derivative of the Helmholtz free energy density with respect to $B$ at fixed electron density $\mathcal{N}$ and temperature $T$, $M=-\left.(\partial F / \partial B)\right|_{\mathcal{N}, T}$. For the present model with the LL's $E_{n, s}\left(y_{0}\right)$, the Helmholtz free energy density is given by

$$
\begin{aligned}
F(B, T) & =\mu \mathcal{N}-\frac{1}{L_{y}} \frac{N_{\nu}}{\beta} \int_{0}^{L_{y}} d y_{0} \sum_{n, s} \ln \left\{1+e^{\beta\left[\mu-E_{n, s}\left(y_{0}\right)\right]}\right\} \\
& \equiv \mu \mathcal{N}-\frac{1}{\beta} \int d E D(E, B) \ln \left\{1+e^{\beta(\mu-E)}\right\},
\end{aligned}
$$

where $\beta=1 / k_{B} T, N_{\nu}=N_{L} / S$, and $\mu$ is the chemical potential. Note that we have defined in the above equation 
the density of states (DOS) per area

$$
\begin{aligned}
D(E, B) & =\frac{1}{L_{x} L_{y}} \sum_{n, k, s} \delta\left(E-E_{n, s}\left(y_{0}\right)\right) \\
& =\frac{N_{\nu}}{L_{y}} \sum_{n, s} \int_{0}^{L_{y}} d y_{0} \delta\left(E-E_{n, s}\left(y_{0}\right)\right),
\end{aligned}
$$

where we have replaced $k$-sum with $\frac{L_{x}}{2 \pi} \int d k$ and used the relation $y_{0}=l_{b}^{2} k$. The explicit inclusion of the DOS in the expression can be utilized to take into account the impurity effect, which broadens the LL's into Gaussian or Lorentzian in shape. For simplicity we did not consider the broadening effect in this paper. In the absence of edge states, the LL's $E_{n, s}\left(y_{0}\right)$ are uniform in space and thus Eq. (15) reduces to

$$
F(B, T)=\mu \mathcal{N}-\frac{N_{\nu}}{\beta} \sum_{n, s} \ln \left\{1+e^{\beta\left(\mu-E_{n, s}\right)}\right\} .
$$

The $B$ dependent chemical potential $\mu$ is connected to the experimentally accessible electron density $\mathcal{N}$ via the local density of states (DOS). In the clean sample limit this is written as

$$
\mathcal{N}=\frac{N_{\nu}}{L_{y}} \int_{0}^{L_{y}} d y_{0} \sum_{n, s} f_{n s}\left(y_{0}\right),
$$

where $f_{n s}\left(y_{0}\right)=\frac{1}{e^{\beta\left[E_{n, s}\left(y_{0}\right)-\mu\right]}+1}$ is the Fermi distribution for the spin-split LL's $E_{n, s}\left(y_{0}\right)$. From Eq. (17) the magnetization density becomes

$$
\begin{aligned}
M & =\sum_{n, s}\left\{-N_{\nu} \int_{0}^{L_{y}} \frac{d y_{0}}{L_{y}} f_{n s}\left(y_{0}\right) \frac{\partial E_{n, s}\left(y_{0}\right)}{\partial B}\right. \\
& \left.+\frac{e}{h} \frac{1}{\beta} \int_{0}^{L_{y}} \frac{d y_{0}}{L_{y}} \ln \left\{1+e^{\beta\left[\mu-E_{n, s}\left(y_{0}\right)\right]}\right\}\right\} \\
& \equiv M^{(0)}+M^{(1)} .
\end{aligned}
$$

One can see that the magnetization consists of two parts. The first part $M^{(0)}$ is the conventional contribution from the $B$ dependence of the LL's and thus denotes a diamagnetic response. The second part $M^{(1)}$ comes from the $B$ dependence of the level degeneracy factor $N_{\nu}$, thus describing the effect of the variation of the density of states upon the magnetic field and denoting a paramagnetic contribution to the total magnetization. Obviously, $M^{(0)}$ is negative while $M^{(1)}$ is positive, the net result is an oscillation of the total magnetization $M$ between the negative and positive values as a function of $B$. At zero temperature, the expression for $M$ reduces to a sum over all occupied LL's:

$$
\begin{aligned}
M & =\sum_{n, s}^{\text {occ }}\left\{-N_{\nu} \int_{0}^{L_{y}} \frac{d y_{0}}{L_{y}} \frac{\partial E_{n, s}\left(y_{0}\right)}{\partial B}\right. \\
& \left.+\frac{e}{h} \int_{0}^{L_{y}} \frac{d y_{0}}{L_{y}}\left[\mu_{0}-E_{n, s}\left(y_{0}\right)\right]\right\},
\end{aligned}
$$
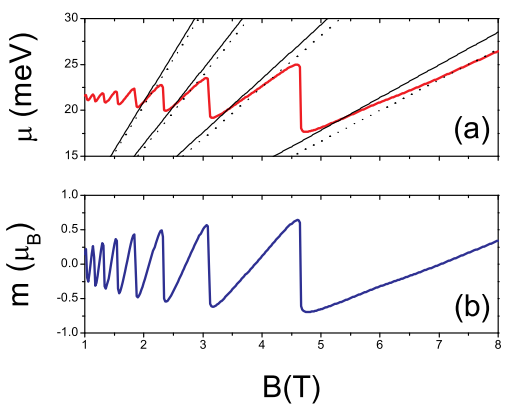

FIG. 2: (Color online) Magnetic field dependence of (a) Chemical potential $\mu$ (thick line) and Landau levels (dashed line: spin down; solid line: spin up) and (b) magnetization $m$ (per electron) for the 2DEG without edge states and spin-orbit coupling. The other system parameters are $\mathcal{N}=4.5 \times 10^{-3} / \mathrm{nm}^{2}, g_{s}=4, m=0.05 m_{e}$, for the inversion heterostructure $\operatorname{In}_{0.53} \mathrm{Ga}_{0.47} \mathrm{As} / \mathrm{In}_{0.52} \mathrm{Al}_{0.48} \mathrm{As}$, and $T=3.5 \mathrm{~K}$.

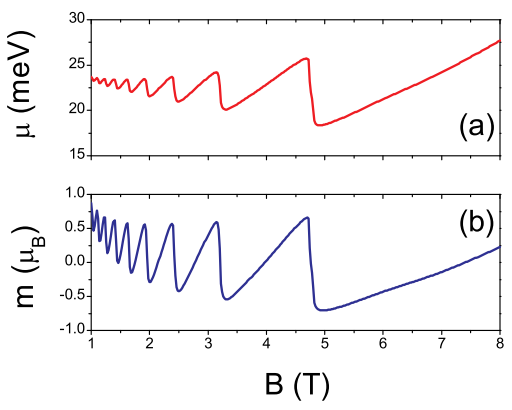

FIG. 3: (Color online) The chemical potential $\mu$ (a) and the magnetization per electron $m$ (b) in units of $\mu_{B}$ in the sample with edges as a function of the external magnetic field $B$. The Rashba coupling $\lambda=0$. The sample size $L=600 \mathrm{~nm}$. The other parameters are the same as those in Fig. 2.

where the sum runs over all occupied states and $\mu_{0}$ is the zero-temperature chemical potential (Fermi energy).

For comparison, let us start from the conventional result for the bulk 2DEG without SOI and edge-state effects. In this case, both the chemical potential $\mu$ [Fig. $2(\mathrm{a})$ ] and the magnetization (per electron) $m$ [Fig. 2(b)] display the well-known sawtooth behavior with varying the magnetic field. At zero temperature, the explanation of the dHvA oscillation can be given with the help of the filling factor $\nu=\mathcal{N} / N_{\nu}$, which measures the number of occupied LL's and is an integer when all the available states in the $\nu$ lowest Landau levels are filled. At these integer values the $2 \mathrm{DEG}$ is incompressible and the chemical potential jumps discontinuously between two adjacent LL's by an amount of $\Delta \mu_{0}=\hbar \omega_{c}=2 \mu_{B} B\left(\mu_{B}=e B / 2 m^{*}\right)$, which defines the incompressibility gap. Note that the abrupt jump in the dHvA oscillation is on the high magnetic field side of the sawtooth, which is special for our present choice of the thermodynamic system. If the system is constrained to have constant chemical potential, 


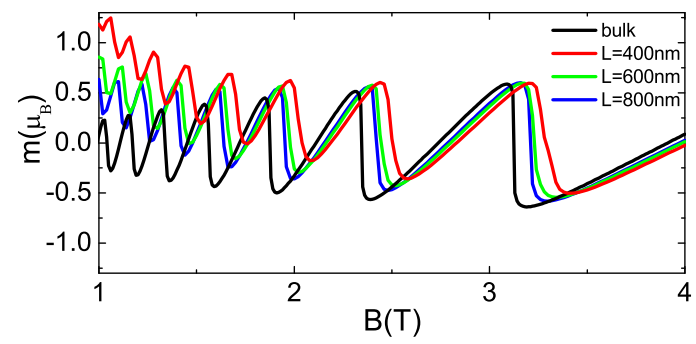

FIG. 4: (Color online) The magnetization per electron $m$ in units of $\mu_{B}$ in the sample with edges as a function of the external magnetic field $B$. The Rashba coupling $\lambda=0$. The sample size $L=400,600,800 \mathrm{~nm}$. The other parameters are the same as those in Fig. 2.

then the jump in the dHvA oscillation will move to the low magnetic field side of the sawtooth, which has been confirmed by Meinel et al. $\underline{\underline{4}}$ in an experiment with the electron density $\mathcal{N}$ modulated by applying a gate voltage to the sample. The zero-temperature behavior of the magnetization curve can be seen by Eq. (20), which in the absence of edge states is

$$
M=\frac{e}{h} \sum_{n, s}^{\text {occu. }}\left(\mu_{0}-2 E_{n, s}\right) .
$$

From above equation, one can easily derive a simplified Maxwell relation $\frac{\Delta M}{\mathcal{N}}=\frac{\Delta \mu}{B}$. Thus the discontinuous jump $\Delta M$ is related to the discontinuity in the chemical potential $\Delta \mu$, i.e., the Landau energy gap at even and the Zeeman gap at odd filling factors, according to $\Delta \mu=\Delta M \cdot B$. When the magnetic field increases within an odd filling factor $\nu$, the Fermi energy $\mu_{0}$ and LL's also increase with $B$. As a result, the magnetization also increases rapidly as a function of $B$, then evolves a maximum at adjacent filling factor $\nu-1$ and suddenly jumps to a negative value. The zero-temperature magnetization jump at these even filling factors are given by the above Maxwell relation, $\Delta M=2 \mu_{B}$, independent of the magnetic field and spin-splitting. At finite temperature as shown in Fig. $2(T=3.5 \mathrm{~K})$, the oscillation amplitude of the magnetization increases with increasing the magnetic field. This fact is due to that the influence of finite temperature in this case (i.e., no SOI and no edge states in the 2DEG) is merely to reduce the oscillation amplitude and the discontinuities in $\mu$ and $M$ via the smearing of the Fermi-Dirac distribution. Another fact revealed in Fig. 2 is that the inclusion of the Zeeman splitting in the LL's does not change the dHvA oscillation modes of the physical quantities. It is due to this fact that the LL's are usually treated to be spin degenerate in previous work.

Now let us see the edge-state effects on the magnetization. Fig. 3 shows the influence of the edge states on the oscillations of chemical potential and magnetization (dHvA oscillations) with magnetic field. The most prominent feature brought by the edge states is that the center of the dHvA oscillations is now dependent on the
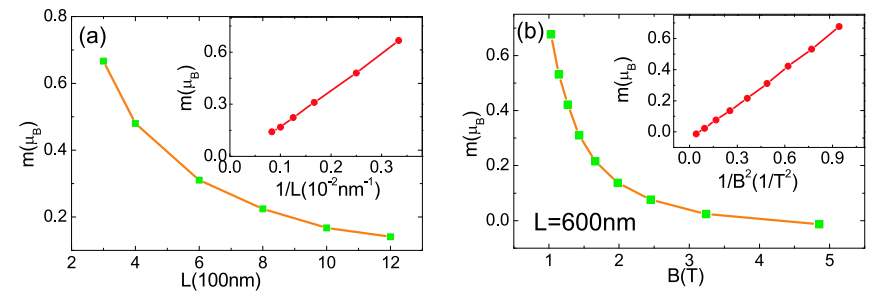

FIG. 5: (Color online) (a) The dependence of the center of dHvA oscillation on the size of the sample. The magntic field $B$ is chosen aroud $1.2 \mathrm{~T}$. (b) The $B$ dependence of the center of dHvA oscillations. In both figures the Rashba SOI is neglected.
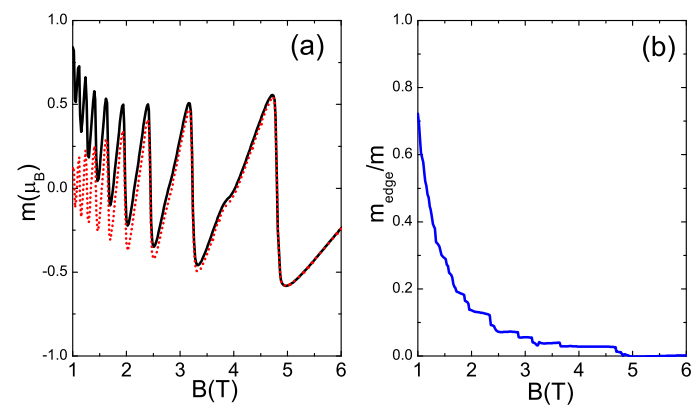

FIG. 6: (Color online) (a) Red curve: The bulk contribution of the magnetization $m$ per electron (in units of $\mu_{B}$ ); Black curve: The total magnetization per electron $m$ (in units of $\left.\mu_{B}\right)$. The Rashba coupling $\lambda=0$. (b) The ratio of edge states contribution of magnetization to the total magnetization. $L=$ $600 \mathrm{~nm}$.

magnetic field. In particular, for the field less than 1 Tesla, the oscillatory magnetization is always positive in sign. Another feature shown in Fig. 4 is that the oscillation amplitude decreases with decreasing the sample size. As is known, the origin of $\mathrm{dHvA}$ oscillations is the degeneracy of Landau levels. The edge states with dispersion lead to edge current which not only is crucial for the quantum Hall effects, but also very important for the magnetization ${ }^{30}$. The dispersion of the edge states partially lift the degeneracy of the Landau levels. Thus the edge states tend to destroy the dHvA oscillations. Therefore it leads to the decreasing of oscillation amplitude as shown in Fig. 4. The upshift of the center of dHvA oscillations may be understood in the following way. For the effects from the edge states, what really matters is the ratio of two important length scales: the magnetic length $l_{b}$ and the system size $L_{y}$. The decreasing of $L_{y}$ is equivalent to the increasing of $l_{b}$, i.e., decreasing of $B$ or $\omega_{c}$. As seen in Eq. (6), in the case with smaller $L_{y}$ or weaker $B$, the second term becomes less important and the eigenenergy is less sensitive to the magnetic field for states with $y_{0}$ near the edges. From Eq. (20), one sees that the second term overcomes the first term and leads to the upshift of the center of dHvA oscilla- 
tions. The smaller the system size is, the more profound effects the edge states lead to, as seen in Fig. 4 for both the center and amplitude of the $\mathrm{dHvA}$ oscillations. Fig. 5(a) shows quantitatively the system size dependence of the shift of the oscillation center. It has the dependence $1 / L_{y}$. Roughly, the contribution of the edge states is proportional to the number of edge states (as also seen from Eq.(20) ), which is proportional to $\nu r_{c} / L_{y}$, where the cyclotron radius $r_{c}=\sqrt{\nu} l_{b} \underline{43}$, with the number of the occupied Landau levels $\nu \sim 1 / l_{b}^{2}$. Thus the center of dHvA oscillations is proportional to $l_{b}^{4} / L_{y}=1 / B^{2} L_{y}$. The $B$ and $L_{y}$ dependence is clearly seen in Figs. 4 and 5 . To see more explicitly the contribution from edge states and bulk states, we plot the total magnetization and the contribution from bulk states in Fig. 6(a). The contribution from the edge states is obtained from Eq. (19) by summing over terms from edge states, with $\left|y_{0}\right|<r_{c}$ or $\left|L-y_{0}\right|<r_{c}$. The rest contribution is from bulk states. There is no upshift of the magnetization oscillation center for the part from bulk states. It shows explicitly that the upshift of the center of dHvA oscillations is due to the existence of edge states. Fig. 6(b) shows the dependence of edge states contribution on the magnetic field. The contribution from edge states increases as decreasing the magnetic field, or equivalently decreasing the sample size as one expects.

When the Rashba SOI is introduced, the filling factor $\nu$ is not linearly proportional to the inverse of the external field $B$, and there is a energy competition between the Zeeman coupling and the Rashba coupling. Also, due to the entanglement between the orbital and spin degrees of freedom, it is difficult to distinguish their separate contributions to the total magnetization. These factors make the physical picture of the $\mathrm{dHvA}$ oscillations to change fundamentally, as shown in Fig. 7(a) for chemical potential $\mu$ and Fig. 7(b) for magnetization $M$ as functions of $B$. One can see from these two figures that the Rashba SOI has no visible influence on the magnetic oscillations of the quantities $\mu$ and $M$ at large values of $B$, where the Zeeman and spin-orbit coupling splitting are small compared to the Landau level splitting. At low magnetic field, however, the SOI modulation of the magnetic oscillations becomes obvious, which can be clearly seen by the enlarged plots of $\mu$ and $M$ in Fig. $7(\mathrm{c})$ and $7(\mathrm{~d})$ respectively for $B$ less than $2.4 \mathrm{~T}$. For comparison, we also re-plot in Figs. 7(c)-(d) the cases without Rashba SOI. One can see from these two figures that the SOI brings about two new features at low magnetic field: (i) The sawtooth structure is inversed, i.e., the location of peaks in $\mu$ and $M$ with SOI correspond to the valleys without SOI. This inversion is due to the different LL's in the two cases. (ii) The oscillation mode is prominently modulated by SOI and a beating pattern appears. This beating behavior in the oscillations are due to the fact that the LL's $E_{n}^{+}$and $E_{n}^{-}$are now unequally spaced due to the presence of SOI.

Another effect caused by the Rashba spin-orbit coupling is that there are weak peaks appearing in the chem-
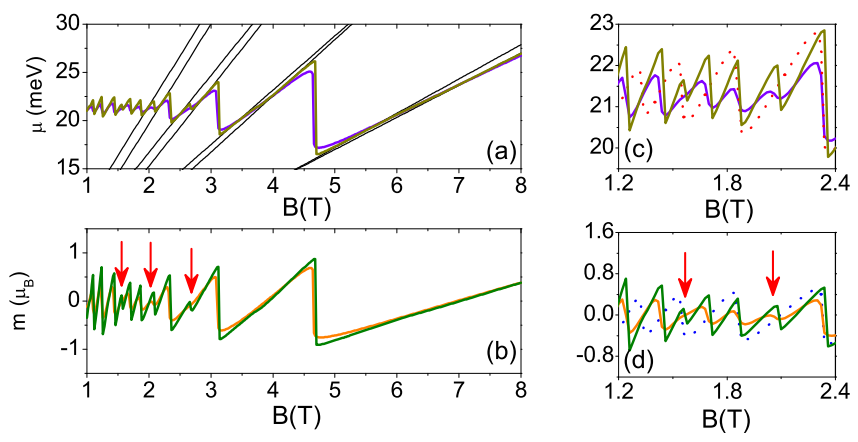

FIG. 7: (Color online) (a) The Chemical potential $\mu$ and (b) the magnetization $m$ (per electron) for the 2DEG under different temperatures $T=3.5 \mathrm{~K}$ and $1 \mathrm{~K}$ with Rasha spin-orbit interaction in the absence of edge states. the Rashba coupling is taken to be $\lambda=15 \mathrm{meV} \mathrm{nm}$. The other parameters are same as those in Fig. 2. The detailed oscillations of $\mu$ and $m$ at low magnetic fields are illustrated in (c) and (d) respectively (solid lines), in comparison with the case of $\lambda=0$ (dotted lines).
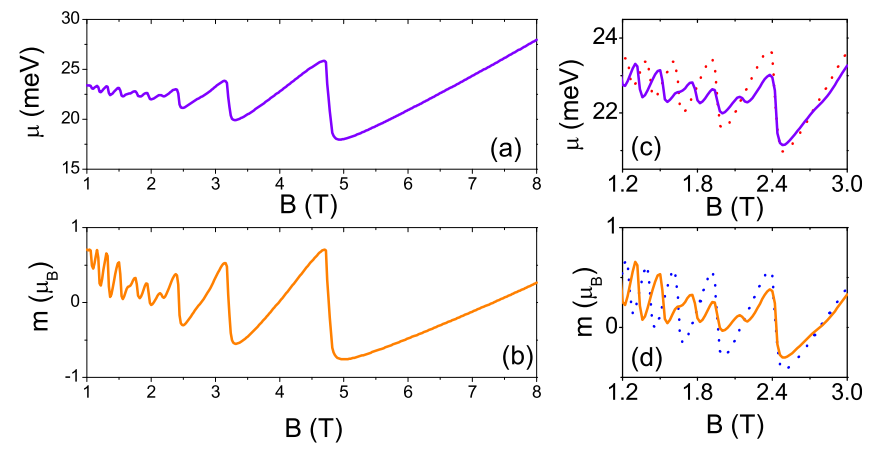

FIG. 8: (Color online) The chemical potential $\mu$ (a,c) and the magnetization $m$ (per electron in units of $\mu_{B}$ ) (b,d) in the sample with edges as a function of the external magnetic field $B$. The Rashba coupling $\lambda=0(\mathrm{a}, \mathrm{b})$ and $\lambda=15 \mathrm{meV}$ $\mathrm{nm}(\mathrm{c}, \mathrm{d})$. In four figures, the sample size $L=600 \mathrm{~nm}$. The temperature $T=3.5 \mathrm{~K}$.

ical potential and the magnetization oscillation at low magnetic field, as shown with the arrows in Fig. 7. In the 2DEG sample without edges, the weak peaks will appear in the magnetization once the temperature is sufficient low, for example, $T=1 \mathrm{~K}$. These weak peaks have been observed recently by Schaapman et al $\stackrel{14}{=}$ when they measured the magnetization of a dual-subband 2DEG, confined in a GaAs/AlGaAs heterojection, and by Zhu et al $\stackrel{10}{\underline{1}}$ when they measured the magnetization of highmobility 2DEG. These peaks is so weak that they will disappear when the temperature is much higher.

Now let us see the SOI effect superposed on the edgestate effect. Figures 8(a)-(b) show the chemical potential $\mu$ and magnetization $M$ respectively in the presence of both SOI and edge states. One can see from these two figures that at large values of $B$, where the Zeeman and 
spin-orbit coupling splitting are small compared to the Landau level splitting, and thus the Rashba SOI has no big influence on the magnetic oscillations of the quantities $\mu$ and $M$. Neither do the edge states, since the cyclotron radius is much smaller than the system size. At low magnetic field, however, both edge states and Rashba SOI change the pattern of dHvA oscillations, as clearly seen in Figs. 8(a), 8(b), and the enlarged plots of $\mu$ and $M$ in Figs. 8(c) and (d) respectively for $B$ less than 2.4 T. For comparison, we also re-plot in Figs. 8(c)-(d) the cases without Rashba SOI. One can see that the total effects are the superposition of effects from both the edge states and Rashba SOI.

\section{MAGNETIC SUSCEPTIBILITY OF 2DEG}

Now we turn to study the magnetic susceptibility $\chi(B)$ for the 2DEG. From Eq. (19), one obtains the expression for $\chi(B)$ as follows:

$$
\begin{aligned}
\chi(B) & =\frac{\partial M}{\partial B} \\
& =\sum_{n, s}\left\{\frac{e}{h} \int_{0}^{L_{y}} \frac{d y_{0}}{L_{y}} f_{n s}\left(y_{0}\right)\left(\frac{\partial \mu}{\partial B}-2 \frac{\partial E_{n s}\left(y_{0}\right)}{\partial B}\right)\right. \\
& -N_{\nu} \int_{0}^{L_{y}} \frac{d y_{0}}{L_{y}}\left[\frac{\beta}{4 \cosh ^{2} \frac{\beta\left[E_{n, s}\left(y_{0}\right)-\mu\right]}{2}}\left(\frac{\partial E_{n s}\left(y_{0}\right)}{\partial B}\right)^{2}\right. \\
& \left.\left.+f_{n s}\left(y_{0}\right) \frac{\partial^{2} E_{n s}\left(y_{0}\right)}{\partial B^{2}}\right]\right\},
\end{aligned}
$$

which at zero temperature reduces to

$$
\begin{aligned}
\chi(B) & =\sum_{n, s}^{\text {occu. }}\left\{\frac{e}{h} \int_{0}^{L_{y}} \frac{d y_{0}}{L_{y}}\left(\frac{\partial \mu_{0}}{\partial B}-2 \frac{\partial E_{n s}\left(y_{0}\right)}{\partial B}\right)\right. \\
& \left.-N_{\nu} \int_{0}^{L_{y}} \frac{d y_{0}}{L_{y}} \frac{\partial^{2} E_{n s}\left(y_{0}\right)}{\partial B^{2}}\right\} .
\end{aligned}
$$

For the ideal noninteracting 2DEG without the edge states, the second term in Eq. (23) is zero due to the fact that both the Landau and the Zeeman splittings of the energy spectrum are linear in $B$. Thus in this case (zero temperature and no edge states) the magnetic susceptibility is simply written as

$$
\chi(B)=\frac{e}{h} \sum_{n, s}^{\text {occu. }}\left(\frac{\partial \mu_{0}}{\partial B}-2 \frac{\partial E_{n s}}{\partial B}\right) .
$$

Furthermore, if the SOI is disregarded, the derivative $\partial \mu_{0} / \partial B$ in quantizing magnetic fields (except at even integer filling factors) is equal to

$$
\frac{\partial \mu_{0}}{\partial B}=\mu_{B}\left[\left(n+\frac{1}{2}\right) \frac{2 m_{e}}{m^{*}} \mp \frac{1}{2} g_{s}\right] \text {. }
$$

Then Eq. (24) clearly shows Landau diamagnetic $(\chi<0)$ at Landau gaps and Pauli paramagnetic $(\chi>0)$ responses at Zeeman gaps.
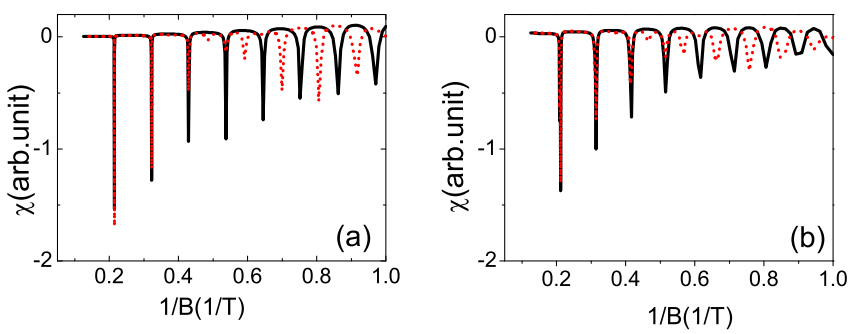

FIG. 9: (Color online) The magnetic susceptibility $\chi$ in the sample (a) without and (b) with edges as a function of the inversed magnetic field $1 / B$. The black (real) line is corresponding to the case of the Rashba SOI strength $\lambda=0$, while the red (dotted) line is corresponding to the case of $\lambda=15 \mathrm{meV} \mathrm{nm}$. In both figures, the electron density and the temperature are respectively set as $n_{s}=4.5 \times 10^{-3} / \mathrm{nm}^{2}$ and $T=3.5 \mathrm{~K}$. The system size in (b) $L=600 \mathrm{~nm}$.

Let us first see the SOI effect on the magnetic susceptibility. Figure $9(a)$ shows $\chi(B)$ as a function of $1 / B$ for the edgeless 2DEG. One can see from Fig. 9(a) that there are a series of equal-distance resonance peaks appearing in the magnetic susceptibility $\chi$ with the magnetic field with/without the Rashba coupling $\lambda$. With the magnetic filed increasing, the magnitude of the resonance susceptibility increases. Similar to that of the magnetization, the explanation of the resonance peaks of the magnetic susceptibility without Rashha coupling also needs the help of the filling factor $\nu \sim 1 / B$. When $\nu$ increases to an integer value, the $2 \mathrm{DEG}$ is incompressible. At this time, all the available states in the $\nu$ lowest Landau levels are filled. Upon increasing the inverse magnetic field $1 / B$, the electrons are transferred to the next Landau level. Thus the chemical potential changes discontinuously (See Fig. 2(a)) and the magnetization jumps (See Fig. 2(b)). Therefore there is a corresponding resonance peak appearing in the magnetic susceptibility $\chi$. When the Rashba spin-orbit coupling is introduced, it will destroy the simply linear relation between the filling factor $\nu$ and the inverse magnetic field $1 / B$. When the value of $1 / B$ is larger, the SOI effect is more evident (See the red line in the Fig. 9(a)).

Figure 9 (b) plots the magnetic susceptibility $\chi$ in a 2DEG sample with edges as a function of the inversed magnetic field $1 / B$. Comparing the black lines in Figs. 9 (a) and 9(b), which corresponding to the case without Rashba spin-orbit coupling, one can find that the resonant (for magnetic susceptibility) magnetic field shifts to larger values for the case with edges. We have obtained the conclusion that the edges not only affect the center of the dHvA oscillation, but also the values of the magnetic field when the magnetization has the discontinuous change. As a consequence, the conditions of the susceptibility (defined as $\chi=d M / d B$ ) having resonance are the same with those of the magnetization. When the Rashba spin-orbit coupling is introduced, similar to the case in the sample without edges, the Rashba coupling changes 
the resonance condition. When the value of $1 / B$ is much larger, the change is much larger as seen in Fig. 9(b).

\section{SUMMARY}

In summary, we have systematically studied the dHvA oscillations of the magnetization and its susceptibility for the 2DEG with the edges states and SOI included in the system.

We find that the edge states and Rashba SOI play important roles when the external magnetic field is small. The edge effect prominently changes the oscillation cen- ter and oscillation amplitude. The dHvA oscillation will change the sawtoothlike form if the Rashba coupling introduced, no matter the sample is with or without edges. The total effects are the superposition of effects from both edge states and SOI.

\section{Acknowledgments}

This work was supported by NSFC under Grants No. 10604010, No. 60776063, No. 10744004, and No. 10874020, and by the National Basic Research Program of China (973 Program) under Grant No. 2009CB929103.
* Author to whom correspondence should be addressed. Email address: zhang_ping@iapcm.ac.cn

1 D. Shoenberg, Magnetic Oscillations in Metals (Cambridge University Press, Cambridge, 1984).

${ }^{2}$ H. Stormer, T. Haavasoja, V. Narayanamurti, A. C. Gossard, and W. Wiegmann, J. Vac. Sci. Technol. B 1, 423 (1983).

3 I. Meinel, D. Grundler, S. Bargstadt-Franke, Ch. Heyn, and D. Heitmann, Appl. Phys. Lett. 70, 3305 (1997).

4 I. Meinel, T. Hengstmann, D. Grundler, D. Heitmann, W. Wegscheider and M. Bichler, Phys. Rev. Lett. 82, 819 (1999).

5 I. Meinel, D. Grundler, D. Heitmann, A. Manolescu, V. Gudmundsson, W. Wegscheider, and M. Bichler, Phys. Rev. B 64, 121306(R) (2001).

${ }^{6}$ F. F. Fang and P. J. Stiles, Phys. Rev. B 28, 6992 (1983).

7 J. P. Eisenstein, Appl. Phys. Lett. 46, 695 (1985).

8 J. P. Eisenstein, H. L. Stormer, V. Narayanamurti, A. Y. Cho, A. C. Gossard, and C. W. Tu, Phys. Rev. Lett. 55, 875 (1985).

9 S. A. J. Wiegers, M. Specht, L. P. Lévy, M. Y. Simmons, D. A. Ritchie, A. Cavanna, B. Etienne, G. Martinez, and P. Wyder, Phys. Rev. Lett. 79, 3238 (1997).

${ }^{10}$ M. Zhu, A. Usher, A. J. Matthews, A. Potts, M. Elliott, W. G. Herrenden-Harker, D. A. Ritchie, and M. Y. Simmons, Phys. Rev. B 67, 155329 (2003).

11 A. J. Matthews, K. V. Kavokin, A. Usher, M. E. Portnoi, M. Zhu, J. D. Gething, M. Elliott, W. G. HerrendenHarker, K. Phillips, D. A. Ritchie, M. Y. Simmons, C. B. Sorensen, O. P. Hansen, O. A. Mironov, M. Myronov, D. R. Leadley, and M. Henini, Phys. Rev. B 70, 075317 (2004).

12 D. R. Faulhaber and H. W. Jiang, Phys. Rev. B 72, 233308 (2005).

13 M. R. Schaapman, P. C. M. Christianen, J. C. Maan, D. Reuter, and A. D. Wieck, Appl. Phys. Lett. 81, 1041 (2002).

14 M. R. Schaapman, U. Zeitler, P. C. M. Christianen, and J. C. Maan, D. Reuter, A. D. Wieck, D. Schuh, and M. Bichler, Phys. Rev. B 68, 193308 (2003).

15 I. M. A Bominaar-Silkens, M. R. Schaapman, U. Zeitler, P. C. M. Christianen, J. C. Maan, D. Reuter, A. D. Wieck, D. Schuh, and M. Bichler, New J. Phys. 8, 315 (2006).

16 M. P. Schwarz, D. Grundler, I. Meinel, Ch. Heyn, and D. Heitmann, Appl. Phys. Lett. 76, 3564 (2000).
17 M. P. Schwarz, M. A. Wilde, S. Groth, D. Grundler, Ch. Heyn, and D. Heitmann, Phys. Rev. B 65, 245315 (2002).

18 M. A. Wilde, M. P. Schwarz, Ch. Heyn, D. Heitmann, and D. Grundler, D. Reuter and A. D. Wieck, Phys. Rev. B 73, 125325 (2006).

19 R. Knobel, N. Samarth, J. G. E. Harris, and D. D. Awschalom, Phys. Rev. B 65, 235327 (2002).

20 J. G. E. Harris, R. Knobel, K. D. Maranowski, A.C. Gossard, N. Samarth, and D. D. Awschalom, Phys. Rev. Lett. 86, 4644 (2001).

21 M. A. Wilde, M. Rhode, Ch. Heyn, D. Heitmann, D. Grundler, U. Zeitler, F. Schäffler, R. J. Haug, Phys. Rev. B 72, 165429 (2005).

22 N. Ruhe, J. I. Springborn, Ch. Heyn, M. A. Wilde, and D. Grundler, Phys. Rev. B 74, 235326 (2006).

23 O. Prus, Y. Yaish, M. Reznikov, U. Sivan, and V. Pudalov, Phys. Rev. B 67, 205407 (2003).

24 A. A. Shashkin, S. Anissimova, M. R. Sakr, S. V. Kravchenko, V. T. Dolgopolov, and T. M. Klapwijk, Phys. Rev. Lett. 96, 036403 (2006).

25 S. V. Kravchenko and M. P. Sarachik, Rep. Prog. Phys. 67, 1 (2004).

26 A. A. Shashkin, Phys. Usp. 48, 129 (2005).

27 S. Anissimova, A. Venkatesan, A. A. Shashkin, M. R. Sakr, and S. V. Kravchenko, and T. M. Klapwijk, Phys. Rev. Lett. 96, 046409 (2006).

28 D. Shoenberg, J. Low Temp. Phys. 56, 417 (1984).

29 F. Oppen, D. Ullmo, and H.Baranger, Phys. Rev. B 62, 1935 (2000).

30 L. Bremme, T. Ihn, and K. Ensslin, Phys. Rev. B 59, 7305 (1999).

31 S. G. Sharapov, V. P. Gusynin, and H. Beck, Phys. Rev. B 69, 075104 (2004).

32 A. Aldea, V. Moldoveanu, M. Nită, A. Manolescu, V. Gudmundsson, and B. Tanatar, Phys. Rev. B 67, 035324 (2003).

33 W.-C.Tan and J. C. Inkson, Phys. Rev. B 60, 5626 (1999).

34 U. Sivan and Y. Imry, Phys. Rev. Lett. 61, 1001 (1988).

35 E. I. Rashba, Sov. Phys. Solid State 2, 1109 (1960).

36 J. B. Miller et al., Phys. Rev. Lett. 90, 076807 (2003).

37 J. Nitta, T. Akazaki, H. Takayanagi, and T. Enoki, Phys. Rev. Lett. 78, 1335 (1997).

38 L. Landau, Z. Phys. 64, 629 (1930).

39 B. I. Halperin, Phys. Rev. B 25, 2185 (1982).

40 A. H. MacDonald and P. Streda, Phys. Rev. B 29, 1616 
(1984).

41 S.-Q. Shen, M. Ma, X. C. Xie, and F. C. Zhang, Phys. Rev. Lett. 92, 256603 (2004).

42 Z. Wang and P. Zhang, Phys. Rev. B 75, 233306 (2007).
43 A. Reynoso, G. Usaj, M. J. Sánchez, and C. A. Balseiro, Phys. Rev. B 70, 235344 (2004). 